\title{
Load Balancing and Relaying Framework in TDD W-CDMA Multi-hop Cellular Networks ${ }^{\dagger}$
}

\author{
Y. Hung Tam ${ }^{1}$, Ahmed M. Safwat ${ }^{2}$, and Hossam S. Hassanein ${ }^{1}$ \\ ${ }^{1}$ Telecommunications Research Laboratory, School of Computing, \\ Queen's University Kingston, ON, Canada K7L 3N6 \\ $\{$ tam, hossam\}@Cs.queensu.ca \\ 2 The Laboratory for Advanced Wireless Networks, \\ Department of Electrical and Computer Engineering, \\ Queen's University, Kingston, ON, Canada K7L 3N6 \\ ahmed.safwat@ece.queensu.ca
}

\begin{abstract}
In 3G cellular networks, high data-rates can be achieved. However, fundamental capacity limitation still exists. Call requests are frequently blocked in hotspot areas. Load balancing among cells helps to solve this problem and to utilize the radio resources. However, existing load balancing schemes are not designed specifically for $3 \mathrm{G}$ networks. Some schemes are not flexible enough or even practical for such networks. In this paper, we propose a novel load balancing and relaying framework for 3G TDD W-CDMA multi-hop cellular networks. This framework consists of a load balancing scheme called ALBA, a routing scheme called ACAR and a slot assignment scheme called E-DSSA. Our framework provides a realistic integrated load balancing solution for these networks. Simulation results show that this framework reduces the call blocking ratios of hotspot areas, balances load among cells and increases system throughput with low packet delay.
\end{abstract}

\section{Introduction}

Mobile communication has recently become affordable and popular. Wireless communications has gone through three generations. In $3 \mathrm{G}$ systems, CDMA is selected as the multiple access technology. A wider band is used to achieve higher data-rates. However, fundamental capacity limitation of these networks still exists. Call requests are frequently blocked in hotspot (congested) areas, such as city centers. Load balancing in a cellular network helps to solve this problem by shifting the load from a hotspot cell to less loaded cells. However, existing proposed load balancing schemes are not designed specifically for $3 \mathrm{G}$ networks such that some schemes are not practical or flexible enough for these networks. For examples, channel borrowing [3] and bandwidth migration [10] for load balancing works in conventional cellular networks, but

\footnotetext{
$\dagger$ This project has been supported in part by Natural Sciences and Engineering Research Council of Canada (NSERC) and Communications and Information Technology Ontario (CITO).
} 
they are not practical for CDMA network because there are no additional channels (frequencies) or bandwidth that can be borrowed or migrated. In CDMA systems, one frequency is reused for all the cells in the systems. Cooperative (coverage) negotiation approach [5] varies the size and shape of cells using antenna technology to achieve load balancing. It is based on the assumption that cell capacity remains unchanged when cell size varies. This assumption does not apply to CDMA systems in which capacity of a cell decreases as coverage increases, i.e. cell breathing effect [7].

Recently, multi-hop relaying in cellular networks has gained attention because it has a potential to increase cell capacity and coverage. It also facilitates load balancing among cells. Load balancing schemes based on multi-hop relaying are Integrated Cellular and Ad Hoc Relay (iCAR) [4] and Pervasive Ad hoc Relaying for Cellular System (PARCelS) [16]. iCAR places low cost limited mobility Ad hoc Relay Stations (ARSs) in hotspot areas for traffic relaying and load balancing. However, iCAR is not flexible enough to handle the highly dynamic load situation of $3 \mathrm{G}$ cellular networks in which hotspots may surface at anytime, anywhere. The dynamic load situation is due to the fact that a large range of data-rates is provided for the users. PARCelS uses mobile nodes for relaying. When a base station (BS) is congested, mobile nodes search best routes to other non-congested cells. Route information is forwarded to BSs for selection. Obviously, the search requires high routing overhead.

Although multi-hop relaying concept is useful for load balancing, it raises routing and medium access issue. These issues could greatly affect load balancing. For Opportunity Driven Multiple Access (ODMA) [6] and Ad hoc-Cellular (A-Cell) relay [14] do not provide a routing solution. Multi-hop Cellular Network (MCN) [11], Hybrid Wireless Network (HWN) [2], Base-Centric Routing (BCR) [8] and PARCelS do not fully utilize the $3 \mathrm{G}$ CDMA systems for routing. In medium access, ISM bands and contention based medium access protocols are usually assumed for the relaying component. These introduce co-channel interference and medium access competition with other non-cellular users.

While a variety of load balancing and relaying strategies were proposed, none of them is perfectly fit into the $3 \mathrm{G}$ environment in terms of relaying, routing, and medium access. In this paper, we propose a novel load balancing and relaying framework for 3G TDD W-CDMA multi-hop cellular networks. This framework integrates load balancing, routing and channel allocation to provide a realistic load balancing solution for these networks. In the next section, we will describe the framework and its components. In section 3 , the simulation model and simulation parameters will be presented. In section 4, simulation results and analysis of the results follows. In section 5, a conclusion and future work will be discussed.

\section{A-Cell Load BAlancing and Relaying Framework (ALBAR)}

We herein propose a novel load balancing and relaying framework called ALBAR for 3G TDD Wideband CDMA (W-CDMA) cellular systems. ALBAR is a centralized dynamic load balancing and relaying framework for consisting of three components: a load balancing scheme called ALBA, a load-aware routing scheme called ACAR, and 
a slot assignment scheme called E-DSSA. All three components are located at the Radio Network Controller (RNC) of a 3G WCDMA cellular network. This framework is designed based on the A-Cell relay architecture [14] in which directive antenna [12] is used. Figure 1 shows the architecture of the framework.

In this framework, ALBA monitors the traffic load condition. When load balancing is needed, ALBA performs load migration planning. ALBA interacts with ACAR to obtain valid route for relaying. ACAR interacts with E-DSSA for finding channels (time-slot code pair) for each node on the route. ACAR replies ALBA with the route finding. ALBA continues to perform load migration until the load in the system is balanced or no further load migration can be done. Then, ALBA sends signal through to the corresponding BSs for updating. The BSs send signals to the corresponding Source Nodes and Relaying Nodes for updating.

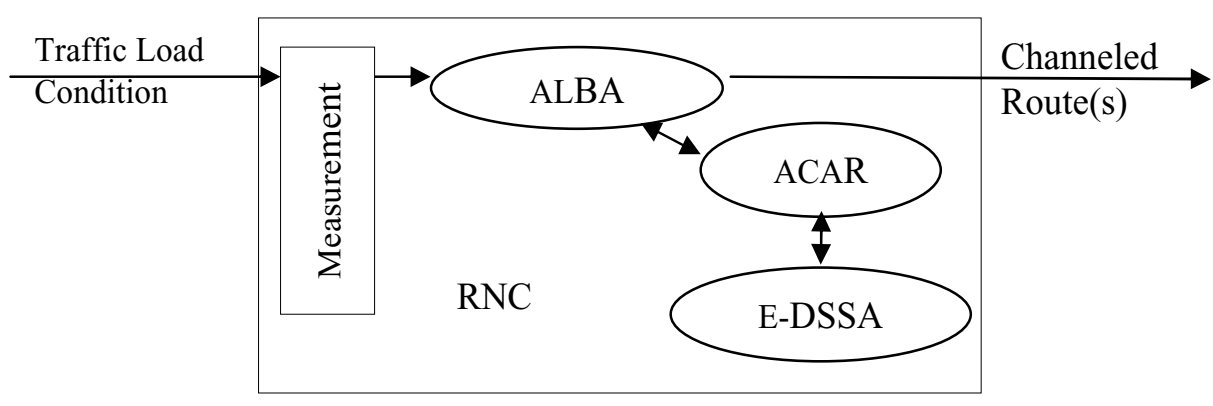

Fig. 1. ALBAR Framework Architecture

\subsection{A-Cell Load Balancing (ALBA) Scheme}

ALBA is a dynamic load balancing scheme for multi-hop ad hoc cellular system. The idea is to shift traffic load from a highly loaded cell to slightly loaded cells in a best effort manner. This manner is used because although source nodes are available for load migration, relaying route may not exist. ALBA is not only suitable for hotspots environment, but also for any heterogeneous load environment. Figure 2 shows the ALBA scheme.

ALBA periodically checks the load status of the networks (Line A1). If Network Load Deviation $\left(D_{N}\right)$ is greater than Network Load Deviation Threshold $\left(D_{\text {NThres }}\right)$, ALBA starts load migration planning. ALBA selects a Target Cell, a Source Cell, and a Source Node of the Source Cell (Line B2 to B5). The Target Cell is the least load cell in the network. The Source Cell is a cell neighboring the Target Cell with the highest load above the Target Cell's load. To proceed further, the Neighboring Load Deviation $\left(d_{n}\right)$ between the Source Cell and the Target Cell must be greater than the Neighboring Load Deviation Threshold $\left(d_{n T h r e s}\right)$ and the Call Blocking Ratio $(C B R)$ of the Source Cell also needs to be greater than or equal to the $C B R$ of the Target Cell; otherwise, no load will be migrated (line B4). Next, ALBA selects a Source Node based on its Migration Priority $(M P)$ level calculated as follows. 
Migration Priority $M P_{i}=k_{1}^{*} C_{i j}+k_{2}^{*} f\left(d_{i}\right)$

Where

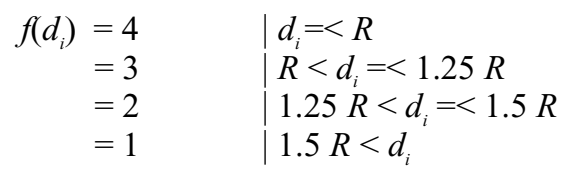

Where $M P_{i}$ is the Migration Priority level of a Connection $j$ of Source Node $i$; $C_{i j}$ is the QoS class of the connection; $d_{i}$ is the distance between the Source Node and the BS of Target Cell; $f\left(d_{i}\right)$ is a mapping function to map the distance $d_{i}$ to a distance factor which is an integer from 1 to 4 ; and $R$ is the cell radius. Constant $k_{l}$ and $k_{2}$ are weighing factors for $C_{i}$ and $f\left(d_{i}\right)$, respectively. The values (1 to 4$)$ of the distance factor are chosen such that the distance factor could break even with the value of quality of service class at some location.

Once a Source Node is selected, ALBA calls ACAR to find a Channeled Route for relaying (Line B6). ACAR replies ALBA with the result of route finding. Whether the result is a success or not, ALBA continues to select another Source Node for load migration until the load of the Source Cell is not greater than the load of the Target Cell or all Source Nodes in the Source Cell are tried (line B7).

If no load can be migrated between the two Cells, ALBA selects the next highest load neighboring cell, higher than the load of Target cell, as the new Source Cell (Line B8). If load migration still fails, ALBA continues to try the next highest load neighboring cell until all neighboring cells are tried or load migration is a success (Line B8). If load migration still fails, ALBA selects the next least load cell as the new Target Cell. If load migration is still a failure, ALBA continues to find a new Target Cell until all cells are tried or load migration is a success.

If load migration between the Source Cell and the Target Cell is a success, ALBA reviews the new load distribution of the network. If the $D_{N}$ is still greater than $D_{\text {NThres }}$, ALBA selects another set of Target Cell, Source Cell and Source Node for load migration (Line B10). These procedures continue until $D_{N}$ is less than $D_{\text {NTrres }}$ or no further load migrations can be done, i.e. all cells are tried. Then, ALBA sends signals through the RNC to the corresponding BSs to update their Channel Allocation Tables and Channels Pools. BSs send signals to the nodes on both old and new routes to update their Channel Tables and Routing Tables.

\section{Illustration of ALBA Scheme}

Figure 3 illustrates how ALBA balances loads in a heterogeneous traffic load environment. First, we choose a Target Cell. Cell C is chosen because it is one least load cell. Then, we choose the Source Cell. Cell A is chosen because it is the highest load neighboring cell of the Target cell C. After three load units are migrated from cell A to cell $\mathrm{C}$, the new load situation is reviewed (see Figure 3b). Load migration continues. Figure $3 \mathrm{~g}$ represents the balanced load situation. 


\section{A) Load Monitoring State \\ 1. Checks the load status of the network through RNC in every Load Sampling Period $\left(T_{s}\right)$ \\ 2. If Network Load Deviation $\left(D_{N}\right)>D_{\text {NThres }}$, then \\ 3. Transit to Load Balancing State}

ALBA Scheme

\section{B) Load Balancing State}

1. Do

2. Do select * (next) least load cell as Target Cell $\left(C_{T r g}\right)$

3. Do select $\dagger$ (next) highest load (higher than $C_{T r g}^{r_{r g}}$ ) non-tried neighboring cell of the $C_{T r g}$ as Source Cell $\left(C_{S r c}\right)$

4. If Neighboring Load Deviation $>d_{n T h r e s}$ and $C B R$ of $C_{S r c}>=C B R$ of $C_{T r}$,

5. Do select non-tried $\operatorname{Src}$ with highest $M P$ for neighboring load migra-

tion.

6. Perform ACAR to find a Channeled Route from the $\operatorname{Src}$ to the $B S$ of $C_{T r g}$.

7. While (load of $C_{S r c}>$ load of $C_{T r g}$ and there are still non-tried $S r c$ )

8. While (neighboring load migration fails and there are still non-tried $C_{S r c}$ )

9. While (neighboring load migration fails and there are still non-tried $C_{T r g}$ )

10. While $\left(D_{N}>D_{\text {NThres }}\right.$ and there are still non-tried $\left.C_{T r g}\right)$

11. ALBA sends signals to corresponding BS(s) through RNC to update their Channel Allocation Tables and Channels Pools.

12. BS(s) sends signals to the nodes on both old and new route(s) to update their Channel Table(s) and Routing Table(s). (Order of signal sending can be simultaneously or least loaded cell first.)

13. Transit to Load Monitoring State.

* - If several least load cells are tied, randomly select one which is not an adjacent cell of previous $C_{\text {rrg }}$ if possible.

$\dagger$ - If several highest load cells are tied, randomly select one of these cells which is not a previous $C_{T r g}$ if possible.

\section{Definition:}

- Source Node $(\boldsymbol{S r c})$ is a mobile node that generates traffic.

- Channeled Route is a route (or path) on which each node can be assigned a Channel.

- Channel Pool is a pool of available channels in the BS.

- Channel Table stores channel information for the connections of Source Nodes.

- Channel Allocation Table stores channel information for the connections in the BS.

- Neighboring Load Deviation $\left(\boldsymbol{d}_{n}\right)$ is the load difference between two neighboring cells.

- Neighboring Load Deviation Threshold $\left(\boldsymbol{d}_{n \text { Thres }}\right)$ is a value above which load migration between neighboring cells can proceed.

- Network Load Deviation $\left(\boldsymbol{D}_{N}\right)$ is the load difference between the highest load cell and the least load cell in the network.

- Network Load Deviation Threshold $\left(\boldsymbol{D}_{\text {NThres }}\right)$ is a value for triggering load migration.

- Load Sampling Period $\left(\boldsymbol{T}_{s}\right)$ is the time interval for checking load of the network.

- Call Blocking Ratio $(C B R)$ is the ratio of number of calls blocked to the number of calls requested. 


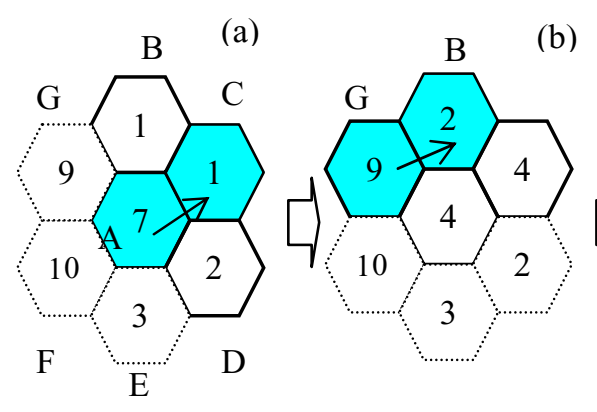

(b)

(c)

(d)
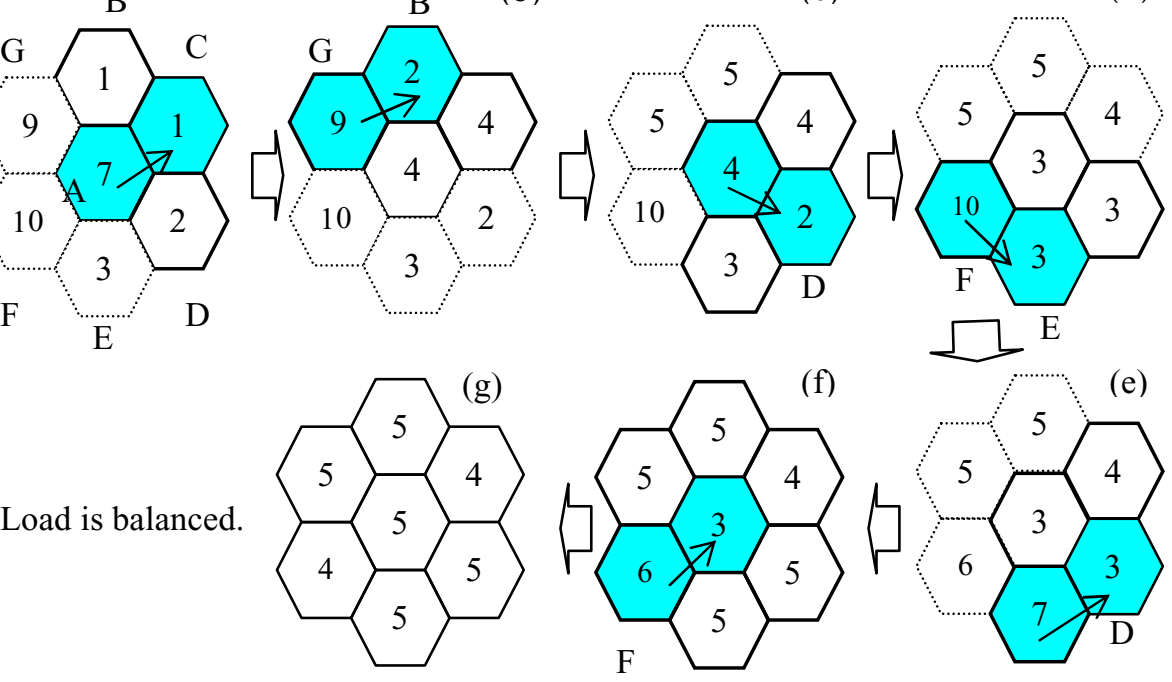

E

Fig. 3. Load balancing of ALBA in heterogeneous load environment

\subsection{A-Cell Adaptive Routing (ACAR) Scheme}

ACAR is a centralized on-demand load-aware routing scheme specifically designed for 3G CDMA cellular systems. ACAR has two mechanisms: Routing Discovery and Route Maintenance. The idea of ACAR is to make use of the cell size flexibility, i.e. the cell breathing effect, of a CDMA cellular system. Route discovery and route maintenance can be done in a single hop with long range and low data-rate while data communication can be done in a multi-hop with short range and high data-rate fashion. In this way, routing overhead can greatly be reduced and the benefits of multihop relaying remain. In addition, no potential call requests, which are within the maximum coverage of a cell, will be denied. Figure 4 illustrates this by comparing MCN-p [11] and ACAR. In MCN-p, since the cell size is shrunk, potential calls from node $\mathrm{A}, \mathrm{B}, \mathrm{D}$, and $\mathrm{E}$ becomes unreachable. In ACAR, these nodes still reach the $\mathrm{BS}$. Thus, no potential call is denied. In Figure $3 \mathrm{~b}$, node $\mathrm{B}$ and node $\mathrm{E}$ are using single hop routing. Node $\mathrm{C}$ and $\mathrm{F}$ are using multi-hop routing. Node $\mathrm{D}$ is using inter-cell routing for load balancing. Details of the scheme can be found in [15].

\subsection{Extended-Delay Sensitive Slot Assignment (E-DSSA) Scheme}

E-DSSA is a slot assignment scheme which is an extension of Delay Sensitive Slot Assignment (DSSA) [1]. DSSA is a slot assignment scheme which is based on A-Cell relay architecture in which directive antennas and Global Positioning System (GPS) is used. In addition to the merits of spatial reuse, channel conflict resolution and the low packet delay attained by DSSA, E-DSSA avoids consecutive channel conflict and 
Last Hop Node Interference. The former is used to avoid signal interference that a mobile should not send and receive data on the same channel at the same time. The latter is used to avoid assigning a channel to a node (current node) such that the Last Hop Nodes on other routes is transmitting on that channel towards the next hop node of that current node. This may happen because the Last Hop Nodes may transmit using Normal Transmission Range $\left(R_{\text {normal }}\right)$ instead of Relaying Transmission Range. Details of the scheme are in [15].

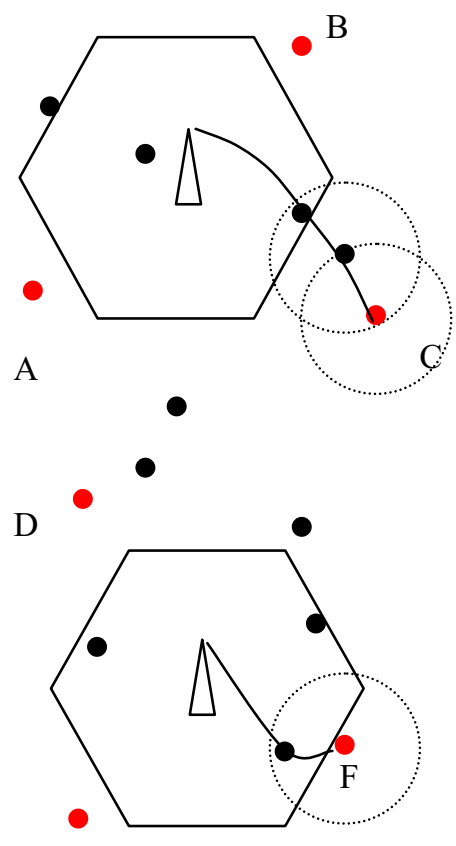

a) MCN-p

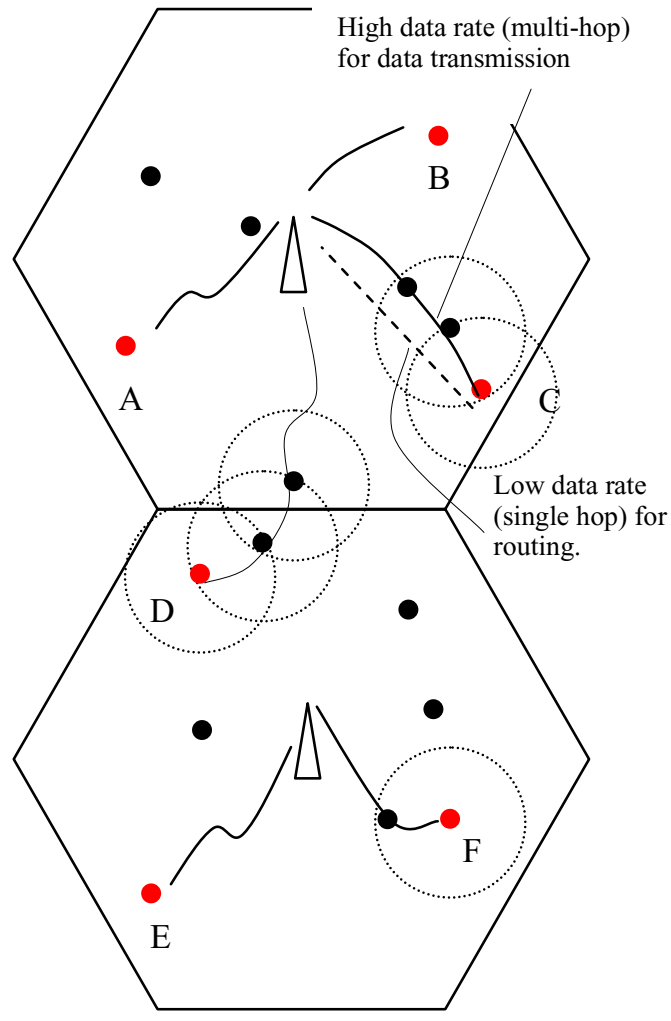

b) ACAR

Fig. 4. Comparison of the routing behavior in MCN-p and ACAR

\section{Illustration of E-DSSA Scheme}

In Figure 5, assume node $\mathrm{C}$ and $\mathrm{B}$ are successfully assigned the channels (slot 5, code 16) and (slot 4, code 2) respectively. Node A is proposed to be assigned a channel (slot 3, code 1). To avoid channel conflict, node $\mathrm{x}$ should not be receiving on this channel and node $\mathrm{z}$ should not be transmitting on this channel; otherwise, channel conflict occurs. In consecutive conflict avoidance, assume a new call request from node D arrives and path D-B-C-BS is the shortest path. Channel (slot 4, code 2) cannot be assigned to node $\mathrm{C}$ for this new path because node $\mathrm{C}$ is currently receiving on 
this channel for the connection of node A. In Last Hop Node Interference avoidance, when a new call request from node $\mathrm{E}$ arrives, node $\mathrm{E}$ can transmit using $R_{\text {normal }}$ because it is the Last Hop Node. Node $\mathrm{E}$ cannot be assigned a channel that node $\mathrm{C}$ is using for reception; otherwise, a channel conflict at node $\mathrm{C}$ occurs. In this case, the channel is ( $\operatorname{slot} 4$, code 2).

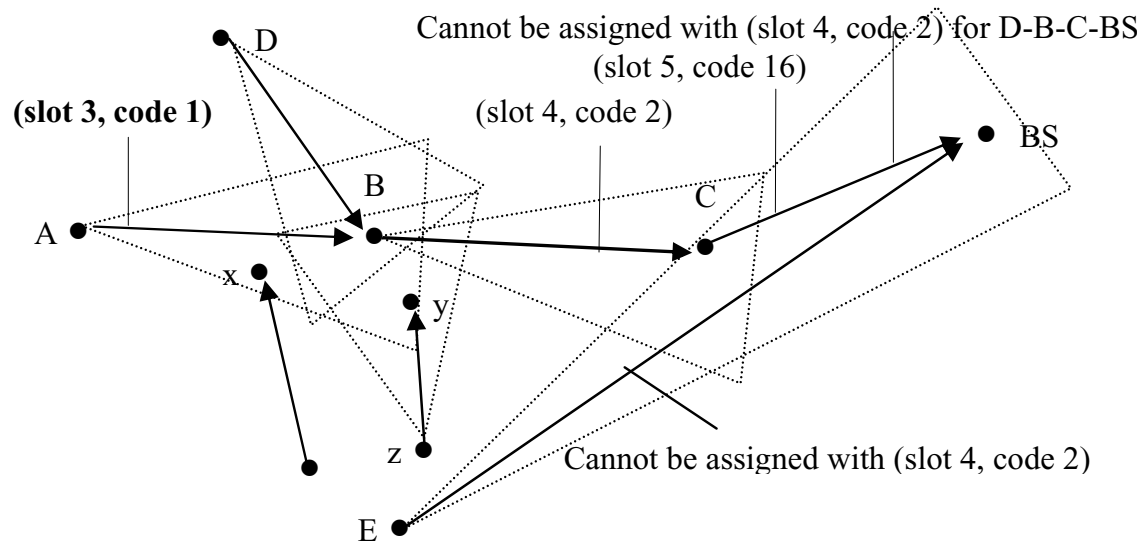

Fig. 5. Conflict and interference resolution of E-DSSA

\section{Simulation Model}

Our simulation model is a three-cell one (see Figure 6). This three-cell model is a generalized model that can be applied to a 3-tier cell system or any geographically limited situation. In fact, this model is a stricter version of a 3-tier cell model because the hot cell has only one neighboring cell for load migration.

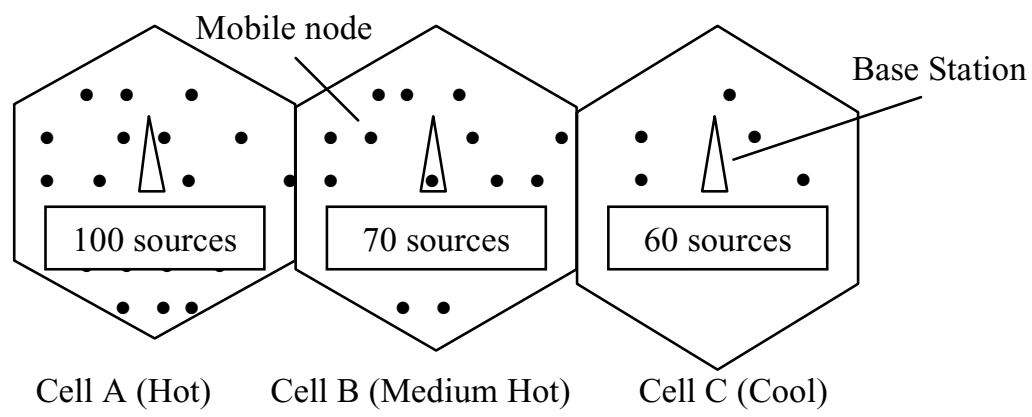

Fig. 6. Three-cell model 


\section{Experimental Setting and Parameters}

In this model, cells $\mathrm{A}, \mathrm{B}$ and $\mathrm{C}$ are respectively hot, medium hot and cool. The numbers of source nodes in cells $\mathrm{A}, \mathrm{B}$, and $\mathrm{C}$ are respectively 100,70 , and 60 . All three cells are connected to the Radio Network Controller (RNC). ALBA, ACAR, and EDSSA are located at the RNC. Each source node generates call requests at an average rate of 0.1 calls/second following Poisson distribution. The average duration of each call is 5 seconds with exponential distribution. The transmission rates for relaying and without relaying are set at $1 \mathrm{Mbps}$. The data rate of each connection is $13.8 \mathrm{kbps}$. Simulation duration is 30 seconds. During the simulation, when a source node places a call request, the call is admitted or rejected by a call admission control (CAC) mechanism. If the call is admitted, CAC calls ACAR to find a channeled route for the connection. The connection can be single or multi-hop depends on the availability of reliable relaying nodes. ACAR chooses a reliable relaying node for relaying based on their state information such as nodal speed, current load and remaining battery capacity. If ALBA is enabled, ALBA checks the load condition of the three cells every 10 seconds. If Network Load Deviation is greater than one connection, load balancing proceeds.

Since the load balancing through relaying is affected by the location of the relaying nodes, we vary the number of relaying nodes in the hot cell or the medium hot cell to investigate the load balancing effect. Two scenarios are formulated as below.

Scenario 1: Relaying nodes in cell A:B:C $=(0 / 40 / 80 / 120 / 160): 160: 120$

Scenario 2: Relaying nodes in cell A:B:C = 160: $(0 / 40 / 80 / 120 / 160): 120$

For scenario 1, while the numbers of relaying nodes in cell $\mathrm{B}$ and cell $\mathrm{C}$ are fixed at 160 and 120 respectively, the number of relaying nodes in cell $\mathrm{A}$ is varied from 0 to 160 at every step 40 . Scenario 2 is similar to scenario 1 except that we vary the number of relaying nodes on cell $\mathrm{B}$ instead while the number of relaying nodes in cell $\mathrm{A}$ and cell $\mathrm{C}$ are fixed at 160 and 120 respectively. The performance metrics used are call blocking ratio of the three cells, load deviation among cells, system throughput, and end-to-end delay of packets under load balancing and without load balancing situation. The OPNET [13] commercial modeling and simulation tool is used. For this simulation, we assume perfect power control, perfect physical medium and slow mobility. We also assume that each relaying node has enough battery capacity. The complete parameter description can be found in [15].

\section{Simulation Results}

The followings are parts of the result of our work. More results are in [15].

\section{Call Blocking Ratio (CBR)}

Figure 7 (scenario 1) shows that, without load balancing, CBR of cell A is approximately 19 to $23 \%$. When the load balancing is enabled, the CBR of cell A drops deeply. The sudden drop, even though there is no Relaying Nodes in cell A, is because there are already many Relaying Nodes in cell B. Once load balancing is triggered, the load of the Source Nodes in cell A which are near to the border of cell B can be relayed immediately to cell B. Then, more capacity is available in cell A. 
Hence, the CBR of cell A decreases. As cell B receives load from cell A, cell B's capacity decreases and its CBR increases. This effect also applies in cell C. This demonstrates the chain reaction of the load balancing effect. When the number of relaying nodes in cell $\mathrm{A}$ increases, the $\mathrm{CBR}$ of cell $\mathrm{A}$ further decreases because more Relaying Nodes in cell A increases the chance for the Source Nodes in cell A in getting routes to cell $\mathrm{B}$.

Figure 8 (scenario 2) shows a similar result except that there is no sudden drop of $\mathrm{CBR}$ even though there are many Relaying Nodes in cell A. The reason is when there is no or not enough Relaying Nodes in Cell B, there is no or not enough relaying route for load migration. Thus, the reduction in CBR in cell $\mathrm{A}$ is relatively smaller. So, the presence of Relaying Nodes in the neighboring cell (cell B) of a hot cell (cell A) provides a faster reaction for load balancing.

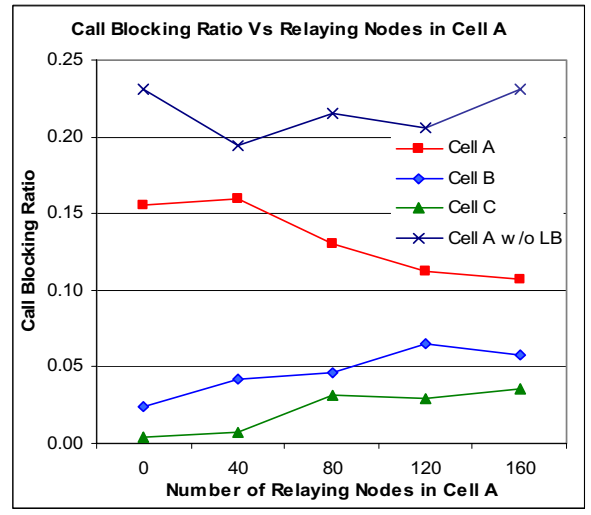

Fig. 7. Call Blocking Ratio among cells with and without load balancing (scenario 1)

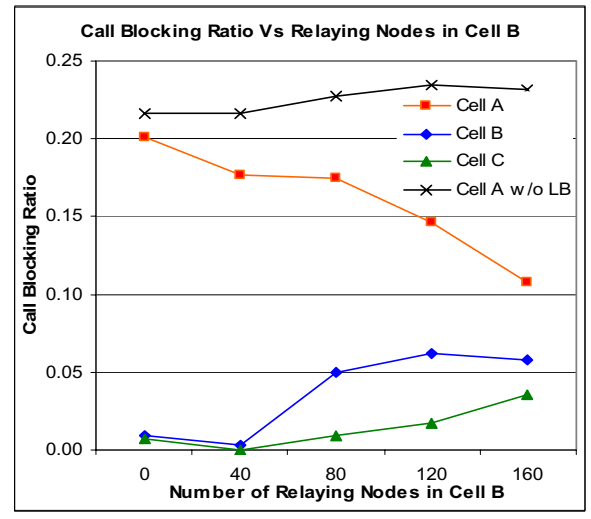

Fig. 8. Call blocking Ratio among cells with and without load balancing (scenario 2)

\section{Load Deviation}

Figure 9 and Figure 10 show that load deviation is reduced in both scenarios when load balancing is enabled. The maximum load deviation, which is the load difference between the highest and the least load cells, follows a similar trend. When the number of Relaying Nodes in cell A or cell B increases, the load deviation is further reduced. This is because more routes are available for load migration. The figures also show that the load deviation drops faster when the number of Relaying Nodes is beyond 40 . The reason is when the number of relaying nodes is sufficiently large, i.e. beyond 40 , there is a higher chance that the relaying nodes fall near to the border of cell $\mathrm{B}$ for relaying. In Figure 10, when the number of Relay Nodes in cell B is beyond 120, the rate of reduction slows down because most Source Nodes has already shifted their load to their neighboring cell. 


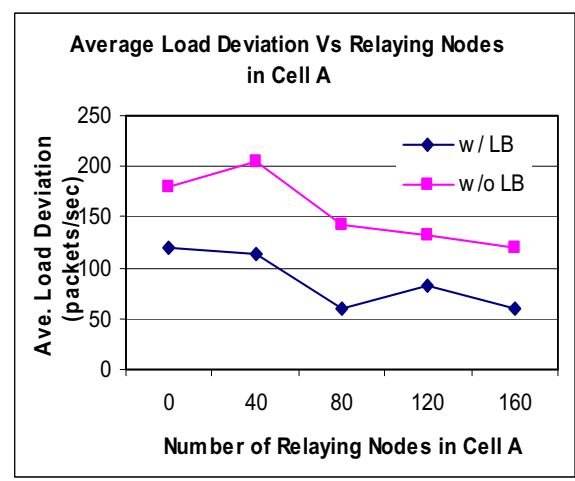

Fig. 9. Average load deviation with and without load balancing (scenario 1)

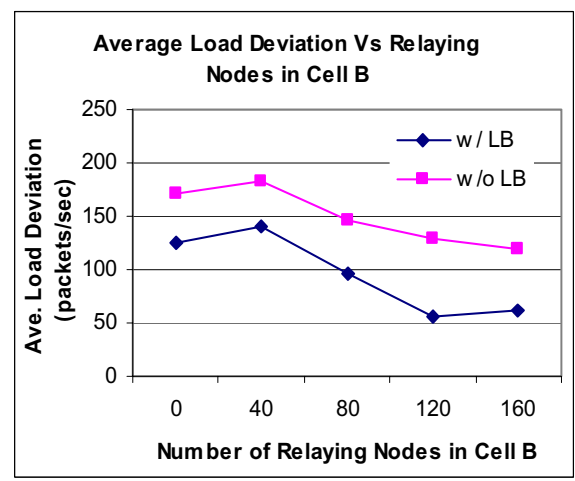

Fig. 10. Average load deviation with and without load balancing (scenario 2)

\section{Throughput}

Both Figure 11 and Figure 12 show that the average BS throughput is increased when load balancing is enabled. Since the load balancing effect reduces call blocking ratio, more calls can be served. Thus, the throughput is increased. Figure 11 shows that the throughput decreases gently when the number of Relaying Nodes increases. This is because the number of hops per route increases. This introduces higher packet delay. Fewer packets arrive at their BSs in same period of time. Thus, throughput is relatively lower. In Figure 12, the throughput does not decrease with the increase in the number of Relaying Nodes in cell B. This is because the Relaying Nodes that are already in cell A dominate the packets' delay. Source Nodes in cell A communicates with the BS using multi-hop relaying from the beginning, increasing the packet delay. Hence, the initial throughput in Figure 12 is lower than the initial throughout in Figure 11.

\section{End-to-End Delay}

Figure 13 shows that the average end-to-end delay of packets is higher when load balancing is enabled. This is because the average number of hops in inter-cell routing is higher than the average number of hops in intra-cell routing. When the number of Relaying Nodes in cell A increases, the delay in both load balancing and without load balancing situations increases. This is because the increase in the number of Relaying Nodes in cell A increases the number of hops in both intra-cell and inter-cell routing. When the number is more than 120, the increase of delay slows down because most routes reach the maximum hop count that has been set. Figure 14 shows a similar result except the delay is lower with load balancing when the number of Relaying Nodes in cell B is around zero. This is because a large number of Relaying Nodes are already present in cell A. The Source Nodes in cell A communicates with the BS of cell A using multi- hopping which causes high packet delay. Therefore, the average delay in cell A dominates the average delay of the system. Thus, a higher delay is obtained even though load balancing is not enabled. When load balancing is enabled, traffic of Source Nodes near to the boundary of cell A starts to be relayed to cell B. 
Since there are fewer Relaying Nodes in cell B at that time, the number of hops in the inter-cell routing is smaller. In other words, the Source Nodes, which are originally using more hops to communicate with their BS, uses fewer hops to communicate with their new BS. Thus, the delay is smaller. When the number of Relaying Nodes in cell $\mathrm{B}$ is beyond 20, the delay with load balancing enabled is higher than the delay without load balancing. This is consistent with the result in Figure 13.

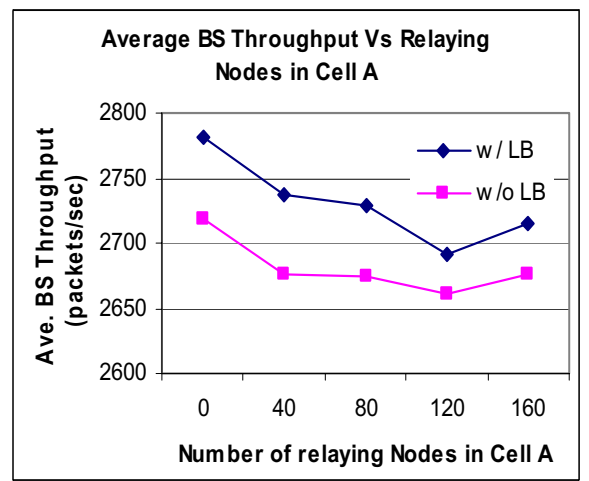

Fig. 11. Average Throughput with and with out load balancing (scenario 1)

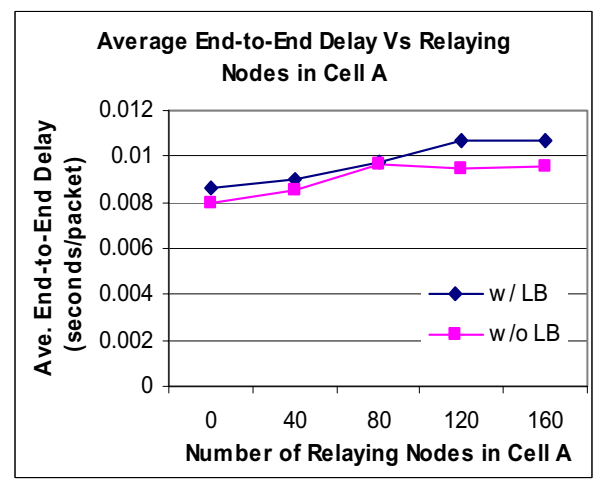

Fig. 13. Average End to End Delay with and without load balancing (scenario 1)

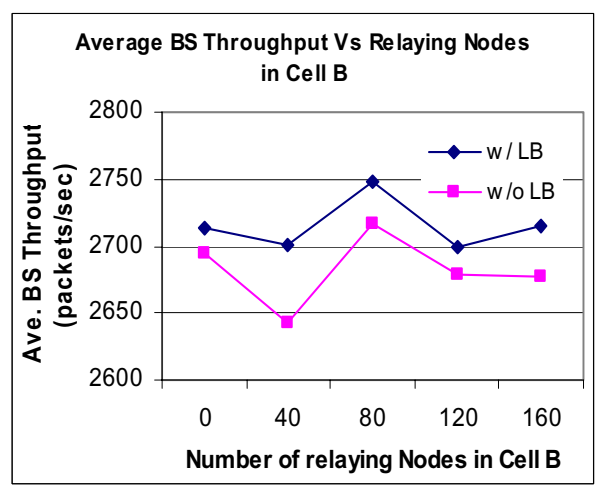

Fig. 12. Average Throughput with and without load balancing (scenario 2)

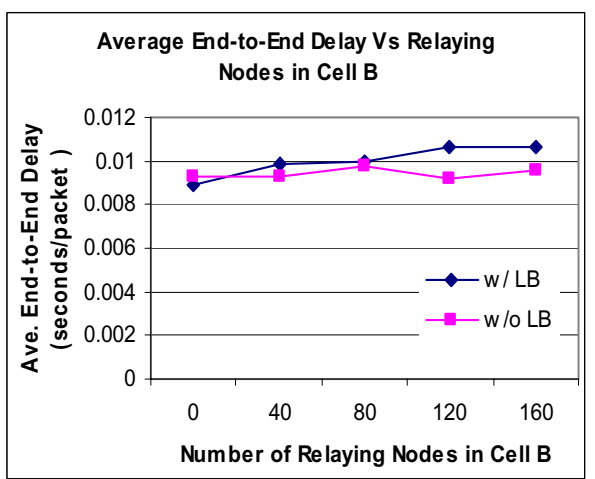

Fig. 14. Average End to End delay with and without load balancing (scenario 2)

\section{Conclusions and Future Work}

A-Cell Load Balancing Relaying Framework (ALBAR) integrates load balancing, routing and channel allocation functionalities to provide a realistic load balancing solution for the 3G TDD W-CDMA multi-hop cellular environment. This framework can be applied to different environments in terms of network topology, load distributions, and relaying route availability. In particular, ALBA can be applied to any het- 
erogeneous load environment. ACAR fully utilizes the dynamic cell size characteristic of CDMA cellular systems such that routing overhead can greatly be reduced and the benefits of multi-hop relaying remain. E-DSSA inherits the merits of DSSA and addresses the dynamic cell size characteristic of CDMA cellular system. Simulation results show that the framework reduces the call blocking ratio of a hotspot cell significantly. Load deviation among cells and maximum load deviation in the network are also reduced. System throughput increases. End-to-end delay of packet increases only slightly. The results also show that the presence of Relaying Nodes in the neighboring cell of a hot cell provides a faster reaction for load balancing.

In the future, optimization of the components of the framework can be performed. A Quality of Service (QoS) provisioning component can also be added to this framework. A detailed comparison of ACAR with other multi-hop cellular routing protocols can be performed. The applicability of this framework to other networks such as mobile ad hoc networks and sensor networks is also an interesting area for study.

\section{References}

1. Al-Riyami, M., Safwat, A. M., Hassanein, H. S.: A Slot Assignment Scheme for TDD WCDMA Multi-hop Cellular Networks. Proceedings of the 22nd Biennial Symposium on Communications, Queen's University, Canada, May (2004) 235-237

2. Chang, R., Chen, W., Wen, Y.: Hybrid Wireless Network Protocols. IEEE Trans. Vehicular Technology, vol. 52. July (2003) 1099-1109

3. Das, S. K., Sen, A.K., Jayaram, R.: A Structured Channel Borrowing Scheme for Dynamic Load Balancing in Cellular Networks. IEEE ICDCS (1997)

4. De, S., Tonguz, O., Wu, H., Qiao, C.: Integrated Cellular and Ad hoc Relay (iCAR) Systems: Pushing the Performance Limits of Conventional Wireless Networks. 35th Annual Hawaii International Conference on System Sciences (HICSS) (2002)

5. Din, L., Bigham, J., Cuthbert, L., Nahi, P., Parini, C.: Intelligent Cellular Network Load Balancing Using Cooperative Negotiation Approach. IEEE WCNC (2003)

6. 3rd Generation Partnership Project (3GPP); Technical Specification Group Radio Access Network; Opportunity Driven Multiple Access. Sophia Antipolis (1999) (3G TR 25.925)

7. Holma, H., Toskala, A.: WCDMA for UMTS, Radio Access for Third Generation Mobile Communications. Revised edition, John Wiley \& Sons (2001)

8. Hsu, Y.C., Lin, Y.D.: Base-Centric Routing Protocol for Multi-hop Cellular Networks. IEEE GLOBECOM (2002)

9. Johnson, D. B., Maltz, D. A.: Dynamic Source Routing in Ad Hoc Wireless Networks. Mobile Computing, Kluwer Academic Publishers (1996)

10. Kim, S., Varshney, P. K.: Adaptive Load Balancing with Preemption for Multimedia Cellular Networks. IEEE WCNC (2003) 1680-1684

11. Lin, Y. D., Hsu, Y.C.: Multihop Cellular: A New Architecture for Wireless Communications. IEEE INFOCOM, Mar. (2000)

12. Ohira T., Gyoda, K.: Design of electronically steerable passive array radiator (ESPAR) antennas. Antennas and Propagation Society International Symposium (2000)

13. OPNET Technologies Inc., www.opnet.com, Sept. (2004) 
14. Safwat, A.: A-Cell: A Novel Multi-hop Architecture for 4G and 4G+ Wireless Networks. Proceedings of IEEE Vehicular Technology Conference (VTC) (2003)

15. Tam, Y. H.: A Load balancing and Relaying Framework in A-Cell Networks. Master thesis, Queen's University, Canada, Sept. (2004)

16. Zhou, J., Yang, Y.: PARCelS: Pervasive Ad-hoc Relaying for Cellular Systems. Proceedings of Med-Hoc-Net, Sardegna, Italy, Sept. (2002) 